Horizons philosophiques

\title{
Philosophie politique sur le mode pragmatico-desperado
}

\section{Robert Hébert}

Volume 10, numéro 2, printemps 2000

Le savoir en fête

URI : https://id.erudit.org/iderudit/802932ar

DOI : https://doi.org/10.7202/802932ar

Aller au sommaire du numéro

\section{Éditeur(s)}

Collège Édouard-Montpetit

\section{ISSN}

1181-9227 (imprimé)

1920-2954 (numérique)

Découvrir la revue

\section{Citer cet article}

Hébert, R. (2000). Philosophie politique sur le mode pragmatico-desperado. Horizons philosophiques, 10(2), 11-24. https://doi.org/10.7202/802932ar d'utilisation que vous pouvez consulter en ligne.

https://apropos.erudit.org/fr/usagers/politique-dutilisation/ 


\title{
PHILOSOPHIE POLITIQUE SUR LE MODE PRAGMATICO-DESPERADO
}

\author{
"Les desperadoes languissent après l'orage, livresse \\ et les blessures... Reprenons l'étude au bruit de l'œu- \\ vre dévorante qui se rassemble et remonte dans les \\ masses". \\ Rimbaud, Les Illuminations, XL1.
}

\section{Vie : actualités 19811}

Quand l'actuelle révolution iranienne bat son plein (d'essence) et actualise les sentiments antiaméricains de tous, applique la loi coranique qui prescrit qu'en cas d'adultère, la femme est mise à mort et l'homme s'en sauve avec 100 coups de fouet alors que cette barbarie explicite notre propre barbarie latente, puisque nous pouvons encore mentalement prononcer cette sentence dans la gestion quotidienne de nos interdits. Et que cette notion pareillement/autrement chrétienne de «foi» fait comprendre qu'une révolution archaïque existe en Islam depuis 12 siècles.

Quand les luttes d'identité polonaises contre l'Ordre Teutonique au $15^{\mathrm{e}}$ siècle font comprendre les résonances géographiques et tardives du mot "patriote», quand ouvriers et paysans polonais persévèrent à mettre en place une autre révolution et qu'il faut les enregistrer avant qu'ils ne soient jugulés-massacrés (comme au massacre des 4,500 officiers polonais à Katyn en 1943, commis au nom de la révolution bolchévique par une police qui n'avait pas encore lu l'Archipel du Goulag).

1. Texte réécrit de la communication présentée au colloque "Comment être révolutionnaire aujourd'hui?" (printemps 1981). 
Quand Bernadette Devlin est laissée pour morte à Belfast en février parce qu'elle représentait encore cette belle folie (incompréhensible) de la révolution irlandaise après 4 siècles de guerre; pendant que 400 prisonniers de l'IRA poursuivent le Dirty Protest depuis 3 ans et que sur les pelouses d'Oxford et de Cambridge, on vit encore le fantasme professionnel et casuistique d'une Revolution in Philosophy.

Quand Ronald Reagan est assermenté sur la Constitution américaine, à Washington, père urbanisé et capitalisé de la Révolution américaine et que l'on sent souffler le vent de la mystique pionnière dans la dénégation pleine du génocide indien qui n'a pas eu le temps de penser sa révolte; que l'on se demande par hasard ce qui est advenu du momentum Black Panther au fil des destins individuels et collectifs. Et puisque la science-fiction guerrière, c'est l'ultime actuel du projet Columbia (il n'y aurait plus de distinction entre réel et fantasme?), "le monde entier nous a vus triompher».

Quand le massacre des ouvriers, des paysans, des étudiants et des prêtres au Salvador, opéré au nom de l'anti-communisme, signale tout un travail de conscience politique entrepris par Simon Bolivar (dont on trouvera une effigie à Montréal, rue Mc Gregor) et dont l'apex fut la révolution cubaine (Che Guevarra traduisant Karl Marx), elle-même comprise par le travail de José Marti, fondateur du Parti Révolutionnaire, tué à la bataille de Dos Rios (1895) et qui n'a jamais traduit, on le devine, Mao Tsé-Toung.

Quand dans cette Italie secouée par les tremblements de terre près de Naples $(3,000$ morts dans l'incurie nationale d'un cauchemar), les secousses terroristes des Brigades rouges ont au moins le mérite fondamental de rappeler un siècle de réflexion politique (indéfiniment différée) dont émerge de ses 10 ans de prison la figure de Gramsci et qui nous rappellent aussi le meurtre de Pasolini. Et telle est la métaphore géologique de la question.

Quand, dans ce pays où est arrivé l'événement le plus important de la civilisation occidentale, la Révolution française, 
surgit l'anti-sémitisme latent des ragots de droite qui disent que la dernière révolution de Mai '68 était dirigée par l'Internationale juive Cohn-Bendit pendant que des agrégés, beaux adolescents ténébreux, ex-tous les istes et les os se convertissent au monothéisme judéo-chrétien et que Louis Althusser assassine Hélène d'une manière peu spinoziste. Et telle est la métaphore psycho-logique de la question, nous faisant réfléchir aux conséquences finales du Principe de Nirvana chez Freud.

Quand Pierre Elliot Trudeau, le plus-que-haineux depuis l'élection du Parti québécois en 1976 («la séparation est un péché contre l'esprit») semonce en janvier les théologiens de la révolution et que nous branlions encore groppusculairement parlant dans le manche de la Révolution tranquille (entre les nationalismes et les socialismes) en oubliant ce que signifie en fait la Loi sur les mesures de guerre. Ou risquant de ne pas comprendre un document dans notre officiel Devoir de fin de semaine qui serait intitulé Révolution des institutions philosophiques au Québec.

Alors tout va bien dans le village global, dans cette «branloire pérenne» comme aimaient le dire les penseurs de la Renaissance. Et cette conjecture d'époque, paroxystique, consiste en ce que l'augmentation de l'information à l'échelle planétaire semble restreindre l'interprétation de l'acte révolutionnaire, alors qu'en fait, se délivre ici-maintenant sa banalité, se livre sa vérité (je ne parle pas de la vraie révolution mais de la vérité de l'acte révolutionnaire).

La révolution technologique a transformé l'Idée même de la révolution. Média d'information permettant autant sa force vive, sa propagation internationale que l'évaluation critique des actes révolutionnaires: nous découvrons des messages que nous ne savions pas lire. Que savons-nous aujourd'hui? de quoi pouvons- nous nous réjouir? Allons-y progressivement. 1) La révolution a toujours été passé simple; elle s'identifie aux archives de la civilisation parce qu'elle s'identifie à la vie, mode de violence réactionnelle à des violences archaïques; et la représentation de la révolution depuis la fin du XVIIIe siècle qui établit une connexion entre une théorie (sociale) de l'homme et 
un projet politique est une modalité fondamentale des révolutions toujours passées. Comment réfléchir cette notion de "modalité" quand le mode ne s'échappe pas de sa cause mais davantage l'accuse? 2) Les révolutions ne manquent pas, au contraire elles abondent; la révolution n'est pas un manque ou l'expression d'un manque qui nous aliénerait à la pensée oiseau-oiseau d'un avenir meilleur ou à l'alibi dévastateur d'une transcendance quelconque; au pire (et c'est là le seul drame à penser), l'acte révolutionnaire se manque et ceci ne nous empêchera jamais d'évaluer-dénoncer les forces de répression, ni d'évaluer les forces de sa trahison quand elle s'oublie dans l'exercice du pouvoir, ni d'évaluer-dénoncer les forces de sa propre annulation (nous savons que l'ennemi du révolutionnaire, c'est l'autre révolutionnaire qu'une étymologie italienne a officialisé : rinnegato - celui dont on dit qu'il nous a renié ou qui dit que nous avons trahi l'idéal commun, ce double sens mériterait réflexion). 3) Le seul sens viable de la révolution réside dans son actualité ici-maintenant parce qu'il n'y a pas de transcendance: préparatifs ascétiques ou somptueux sans fin, gestes et exemple nous-mêmes par où, quotidiennement, communautairement, nous écartons toute forme d'exploitation et d'oppression entre nous; par ailleurs, cette pensée que la révolution, la Grande révolution, est avant tout momentum et parce qu'elle aura été momentum, parce que l'acte révolutionnaire investit l'actuel par des interprétations de révolutions passées, elle sera encore et nous pourrons toujours la penser ici-maintenant.

À la question donc "Comment être révolutionnaire, aujourd'hui?", je répondrai sereinement, c'est-à-dire sur le mode pragmatico-desperado : «il suffit semble-t-il de l'être..." Mais comment? en s'organisant puisque cette métaphore est à l'origine même de l'acte révolutionnaire et donc de la modalité la plus fondamentale de la vie. S'organisant, non seulement en organisant un savoir-faire militant (par exemple, parce que je les crois plus efficaces, sur le modèle des Organisations communautaires qui réussissent patiemment les petites révolutions), davantage en complétant l'idée d'une révolution 
permanente par l'idée d'un dire permanent qui puisse faire savoir le mobile de l'acte révolutionnaire. Grandes ou petites révolutions. Simplicité désarmante de ce mobile : liberté (sociale) sans exploitation ni oppression d'aucun ordre que ce soit, accomplissement (sans exploit) du désir, achèvement (pour tous et pour toutes) de la violence.

\section{Révolution des signes révolutionnaires}

Alors tout commence mal dans le village global; la simplicité engendre, semble-t-il, les armatures les plus complexes.

- Monsieur, vous êtes absolument hors de la question militante et votre position est propre à mêler toutes les cartes, indistinctement. Au nom d'une sorte de télescopage simultanéiste et pseudo-causaliste "pendant que...", "parce que...») que précisément nous critiquons. D'un côté, votre sophistique semble promouvoir l'acte révolutionnaire mais en rendant équivalentes toutes les révolutions - ce qui fait de vous un contre-révolutionnaire. De l'autre côté, votre sophistique semble promouvoir avec cynisme une sorte de nécessité métaphysique de la violence qui au fond masque bête- ment un humanisme classique à partir de quoi vous pointez du doigt le réel, comme si vous ne vouliez pas y participer. Ce sont là de vieilles techniques de conversation petite-bourgeoise qui ne sauraient échapper à notre vigilance.

- Mais non, Je ne mêle pas les cartes, indistinctement; au contraire, je veux bien distribuer les cartes et en montrer le matériau réglé; le langage, l'événement historico-social que nous fabriquons tous et toutes, enfin ce résidu de la vie qui s'appelle "désir» et que la pensée peut indéfiniment analyser et interpréter. Je veux simplement penser l'origine de la question (c'est-à-dire y répondre), afin d'amorcer une réflexion politique car il me semble que la question "Comment être révolutionnaire, aujourd'hui?" devrait être remplacée par celle-ci : "Comment devenir politique, aujourd'hui, sans être dans la représentation d'une politique réussie et instituée?» Je veux donc mettre à l'épreuve un certain savoir-faire nommé philosophie et faire savoir une triple évidence par où une triple 
désaliénation de la question serait actuellement possible. Allons-y progressivement.

Première évidence. II y a dans la question (et dans les textes-documents qui ont circulé autour du colloque) une sorte de dépression épistémique qui, oscillant entre l'hébétude d'avoir entretenu l'idéal révolutionnaire et l'hébétude d'avoir entrevu l'échec des révolutions sur ce plan de l'Idéal, nous atterre par une innocence pour le moins suspecte. Air de post-deleuzisme déterritorialisant qui dans ses mauvais jours magistraux métaphorise (du rhizome au devenir-animal) la déconfiture de la nouvelle philosophie. A-t-on perdu la tête d'avoir trop cru? Ne resterait-il donc que le cœur au ventre mais que faire? de ce ventre qui est toujours un peu aveugle à son élasticité historique? Bien que les actes révolutionnaires soient institutionnalisés et saturés dans leur discours, l'innocence ne saurait être une nouvelle raison d'y déplacer l'attention ou d'en raturer l'acquis; nous ne pouvons pas faire l'économie du processus par lequel s'instaure le vouloir-redevenirinnocent; auquel cas, il faut penser cette dépression. Comment suscite-t-elle des courants de pensée? Comment prend-elle même le masque de la jubilation et de tous les nouveaux lyrismes? Mais surtout, comment ne plus être dans la dépression et néanmoins se réjouir de ce qui semble impossible? sans régresser? En termes hégéliens, je dirais que le dépassement du paroxysme actuel passe par une double reconnaissance : reconnaître que le mauvais infini de la révolution, c'est sa réussite parce qu'aussitôt passé son momentum et sa mise en place politique, c'est-à-dire sa représentation, elle s'offre à l'interprétation et à sa propre critique (suscitant dans son enjeu même d'autres actes révolutionnaires antagonistes); et simultanément, reconnaître que le bon infini de la révolution, c'est son échec parce qu'alors elle sécrète son mobile et son moteur, elle se réactualise comme ayant été actuelle et donne raison de maintenir cette simplicité entre temps armée du mobile révolutionnaire. Et puisqu'il n'y a pas de transcendance, tel est le va-et-vient qui ne finit pas de recommencer - ce que certains appelleraient «dialectique». 
Contre cette dépression flairée dans la question "Comment être révolutionnaire, aujourd'hui?" ", question à laquelle tous les savoir-faire militants répondent pour eux-mêmes, en connaissance de cause, tout donc doit se poursuivre. Et se poursuivra de toutes les façons. La conséquence immédiate de cette position, outre qu'elle dissout des attitudes comme l'humanisme des fins ou le cynisme des moyens, est de promouvoir le polymorphisme des actes révolutionnaires en tant que pulsion de vie. Que ce polymorphisme soit aussi celui de toute violence primitive? bonne ou mauvaise? entre temps, aucun décret puisqu'il n'y a pas de transcendance. Seulement la possibilité de la penser - en décrétant la nécessité d'analyser et de critiquer ce que cette violence tout à coup fonde au nom d'une certaine transcendance. Et ceci renvoie au geste inaugural de ce que la tradition appelle la philosophie politique.

Deuxième évidence. Qu'une telle question se pose à l'intérieur de cette salle, cet espace architectural clos malgré ses entrées et donc ses sorties (ou vice-versa), ce Dedans institué et connoté par un département de philosophie, a quelque chose d'un peu obscène. A de quoi faire grimacer les anges gardiens les plus zélés. Que faire? oui; et que faisons-nous ici, maintenant? D'une part, la révolution a peu besoin de philosophes-penseurs-intellectuels en tant que tels, organiques ou non (ce que dans leur routine bouche à bouche, porte à porte ont compris les ultra-gauches marxistes-léninistes et leur anti-intellectualisme en est symptomatique); peut-être découvrirons-nous en même temps que la révolution a peu besoin de militants en tant que tels, mécaniques ou non. Et je dis cela, non pas pour reprendre l'argument dépressif par excellence - la vraie révolution, c'est le vrai sang, la vraie bataille ailleurs alors que sur le territoire québécois nordaméricain, l'on devrait se contenter d'un vampirisme symbolique entre nous; à tous les niveaux de petites révolutions opérées par les organisations de type communautaires militantes, le travail du silence et de l'exemple ne sont pas des manières moins dignes de lutter contre l'exploitation et l'oppression quotidiennes. D'autre part, la question qui se pose 
ici-maintenant se pose et se repose indéfiniment à l'intérieur des savoir-faire militants : ce qu'on appelle le moment autocritique (dans un petit appartement secret, architecture close) où l'on évalue, on planifie, on distribue des tâches, etc. Autrement dit, le seul intérêt à demeurer ici-maintenant, c'est de faire dévoiler cette métaphore collégiale du va-et-vient entre la pensée et l'action; les doubles passages entre la pensée révolutionnaire et l'action révolutionnaire. Apories classiques titre courant de livre de philosophie, plutôt scolaires. Espace architectural donc qui métaphorise le destin de la question, tellement et si bien que nous risquons de sombrer dans une dépression encore plus profonde; celle de devoir répondre (quoiqu'il en soit de l'acte révolutionnaire) dans cette salle alors qu'il faudrait déambuler indéfiniment et nonchalamment dans la rue. Dehors fantasmé de chaque Grande révolution, en chantant nos discours ou en clamant la bonne nouvelle, ne serait-ce que la question est posée ici, par ce vendredi-samedi du printemps 1981. Mais nous savons que toute révolution (en son désir), qu'elle soit violemment réprimée ou qu'elle réussisse par l'occupation des espaces maintenant vacants, s'achève un jour par le décret de quelque couvre-feu. II faudra retrouver les mêmes espaces asilaires. Usines, écoles, prisons aux architectures ternes, l'appartement familier ou un bar quelconque où l'on scandera, aux petites heures, un chant fraternel et bouleversant. Action du chant et pensée de l'inassignable sens, toujours.

Tout compte fait, la difficulté d'analyser le rapport penséeaction, d'en suspendre le passage comme lieu et source du problème, peut être levée par des simulacres et je crois que ces simulacres représentent les tentations extrêmes (identiques?) qui bornent l'acte révolutionnaire mais que tous les révolutionnaires doivent dénier. Simulacres que je construirais à partir d'une métaphore bien connue: se jeter dans le feu de l'action sans jamais réfléchir ou sombrer dans le feu de la pensée sans nulle part intervenir. Bornes équivalentes? Comment cette pulsion de vie que représente les actes révolutionnaires peut-elle composer avec cet espace architectural? 
Sommes-nous ici-maintenant soumis à l'action où à la pensée, ou au passage secret ou à rien de tout cela s'il s'avérait que l'acte révolutionnaire est trop fondateur et par contre, trop fondamental en son absence, pour être dicible ici même?

Troisième évidence. La transcendance du «nous y arrivons, nous y sommes" étant un leurre nécessaire, nous sommes soumis à la loi de la contradiction sur le terrain même de la question. Bataille nécessaire des interprétations entre interprétants. Loi de la contradiction, non seulement celle promue comme principe général ou descripteur précis lorsque tel projet révolutionnaire construit sa propre épistémologie (matérialisme dialectique) mais surtout celle qui oblige à se définir ou à articuler sa différence par rapport à d'autres projets révolutionnaires, à repérer ou à contrer les moindres différences (voir le double thème de l'ami extérieur l'ennemi parmi nous), à être soi-même l'objet-cible de la loi de la contradiction. Cycle que toutes les organisations militantes style faucon, ont parcouru, parcourront encore entre les luttes intestines réelles (et parfois blessantes, lorsque le cycle s'achève aux chaînes de bicycle) et l'exhortation stratégique, morale, donc fictive, à l'unité transcendante d'un front. Tout ceci est connu; je m'excuse d'être à ce point banal. Mais cette banalité, en ses conséquences lumineuses, laisse entrevoir le travail complexe du rapport entre la pensée révolutionnaire et l'action révolutionnaire: comment la loi de la contradiction (pour être plus juste, de l'antagonisme) exprime la transmutation de cette pulsion de vie que représentent les actes révolutionnaires en une pulsion de mort, sa propre mort en tant que l'ennemi nous ressemble à tous les moments où il prétend aux mêmes objectifs ultimes; comment l'emploi du temps révolutionnaire doit se fractionner et donc s'affaiblir entre un temps où l'on travaille effectivement sur le terrain de la désaliénation et de la désexploitation des humains, et un temps où l'on investit une grande part d'énergie à se contre-définir, à élaborer des plans d'action et de discours contre...; comment la loi de l'antagonisme peut transmuer cette pulsion de mort en un momentum paroxystique qui devient sa pulsion de vie la plus élevée en sa mort, la plus croyante et 
aveugle. Ce moment, c'est celui de l'auto-dissolution "comme un des beaux-arts sociologiques" (René Lourau). L'exemple québécois auquel je renverrais - le numéro 17 de la revue Stratégie (automne 1977) dont la collection complète est soldée au Palais du Livre (le Palais du Livre comme destin de l'Idée révolutionnaire?). Relire les divergences de Stratégie avec Champs d'application qui s'est dissout pour rallier En lutte!, décoder ce complexe auto-évaluatif judéo-chrétien entre Stratégie, En lutte! et la Ligne communiste $(m-1)$ du Canada qui portait bien sûr sur la question disputée d'une politique culturelle marxiste-léniniste. Se rappeler les divergencesdissensions accusées par Chronique (lire le premier numéro en sa déclaration désirante et le dernier numéro) et entre temps essayer de comprendre la circulation extraordinaire des signatures au sein des divers comités de rédaction.

Cette troisième évidence souligne que la prolifération et les paroxysmes les plus prenants dérivent de la loi de la contradiction, dévoilent, ont un sens décodables. Possibilité, émulation des actes révolutionnaires et en même temps (pour cette même raison) leur clôture: comme ici-maintenant, l'échec évident de ce Colloque, c'est qu'il ne manque pas de réponses ou de discours moqueurs, blasés, sérieux, agressifs, que ce plein, doxographique de positions politiques diverses-antagonistes est parfait en ce qu'il détermine déjà notre ici-maintenant; par ailleurs, cet échec sera sa réussite en tant qu'il aura été actuel, mieux encore en tant qu'il donnera raison à tous les savoir-faire militants qui revendiquent la bonne réponse de poursuivre avec plus d'ardeur et de conviction, la multiplicité des interprétations renforçant l'interprétation déjà là des invités interprétants que nous sommes.

\section{Penser: l'actuel}

Alors, tout irait pour le mieux dans le village global?

- Monsieur, vous êtes absolument hors de la question militante, je le répète, et il n'y a rien à faire avec ce savoir-faire que vous appelez "philosophie politique». Par exemple, la troisième évidence que vous avez soulignée n'est pas 
importante; vous ne semblez pas distinguer ce qui est principal et ce qui est secondaire dans la loi de la contradiction que vous interprétez comme une pulsion de mort. Partout aujourd'hui, à l'échelle planétaire, il y a augmentation de la conscience révolutionnaire et luttes réelles; cela suffit. Votre philosophie politique sur le mode pragmatico-desperado tient du délire métaphysique et me donne envie de faire un nouveau colloque d'ici quelques semaines sur le thème "Comment ne pas devenir philosophe, aujourd'hui?».

- Mais non. Tout est plus simple et plus désarmant à la fois. Reposer la question en terme d'une philosophie politique (je n'ai voulu que penser l'origine de la question, c'est-à-dire y répondre) n'annule pas, n'annulera jamais, ce qui se fait, ni les multiples savoir-faire militants qui actualisent et revendiquent l'acte révolutionnaire comme une propriété close. Soyons généreux, la philosophie comprend tout cela. Cette philosophie par conséquent ne préjuge pas, elle recueille; elle promeut la nécessité de la vie en comprenant que chaque acte révolutionnaire en tant que pulsion (commençante) de vie doit aboutir à son terme mais que ce terme se révèle être le processus actuel de son essence. Le mode pragmatico-desperado en philosophie poli- tique, c'est ce qui justement situe l'autre actualité de toute entreprise philosophique. a) Tirer les conséquences de la prolifération des interprétations et de l'antagonisme moteur qui les porte sur le champ de bataille, en termes d'une dialectique qui sait - se discourant dans la conservation de son essence - qu'elle s'offre elle-même au processus indéfini de l'interprétation. b) Penser l'actualité et l'actuel de tout acte révolutionnaire en dehors de toute Intention, de tout régime de Transcendance, de tout leurre utopique - en tant qu'il est nécessaire et suffisant à ce moment-là qu'il se déploie. c) Mode de penser pragmatico-desperado, c'est-à-dire ontologiquement ironique, libéré de l'espoir et du désespoir psychologiques; souriant de savoir que se jeter dans le feu de l'action ou sombrer dans le feu de la pensée (là ou ici, ou à l'imparfait entre-deux sans trop comprendre les effets immédiats) est au fond le secret de tous, et que tout 
savoir-faire n'a d'intérêt que d'actualiser sa propre loi pour faire être ce qui n'était pas.

Travaillant cette question "Comment être révolutionnaire, aujourd'hui?", peut-être aura-t-il fallu transformer notre propre rapport à la philosophie, à l'histoire et à leurs lieux communs avant d'apporter des réponses "concrètes ou délicieuses". Mais transformer quoi au juste? lorsque: sur le territoire québécois notre perception des philosophies ou des histoires contextuelles tient encore d'un néo-colonialisme angélique, d'une sénilité non questionnée, délégation rinnegato d'une question que nous nous posons depuis vingt ans. Depuis les années '70 pourtant, un certain momentum paroxystique - tant au niveau guerrier et politique que poétique et culturel at large - nous habiliterait à opérer cette révolution dans notre rapport archi-institutionnel à la pensée. Et ceci commencerait par "penser ces paroxysmes": première et dernière (toute différente, ratée) Nuit de la Poésie, 1970-1980; première et dernière élection (moins coulante de champagne) du Parti québécois, 1976-1981; Événements d'Octobre et Référendum sur la question nationale, 1970-1980 — dans l'étonnante torpeur silencieuse des semaines qui les ont suivis.

Un jour, quand nous aurons le courage d'analyser l'avènement de la philosophie dans la civilisation (ses conditions socio-rituelles, politiques, langagières, etc.), nous développerons cette interprétation par laquelle la philosophie (c'est-à-dire la pensée du devenir politique) est un moment paroxystique qui répond, en négatif et en creux dans l'espace du discours humain, à d'autres paroxysmes : polémos multiple sur un terrain et pour cette raison, controverse et agonistique sur le terrain même de la pensée et de l'action. Les classiques se révéleraient toujours actuels, à condition d'en forcer le contexte territorial, de présenter quelques règles précises de décodage. Relire bien sûr La République de Platon en biais avec l'inavouable avoué de la Septième lettre; lire l'Éthique à Nicomaque et le Politique du métèque Aristote en biais avec son Grand Recueil des Constitutions - par où, analysant 159 
constitutions. Aristote «répétait» nos gestes d'inventaire quand nous nous penchons sur des documents officiels, juridicopolitiques, et que nous cherchons à comprendre; nouveaux impérialismes de la Russie et des U.S.A., Chine de l'anthologie Révo cul dans la Chine pop, ...l'Albanie. Ou plus près de notre modernité, relire Le Prince d'un Machiavel qui a photographié pour nous "ces sortes de pierres d'attente qu'une révolution laisse toujours pour en appuyer une seconde" (section II, tr. Périès) en biais avec ses Discours Sur la première décade de Tite-Live; lire à Amsterdam les Autorités théologique et politique de l'excommunié-ostracisé mais néanmoins très doux Spinoza (premier penseur de la démocratie) en biais avec l'Éthique et se demander par quelles filières, quels filons le corps de cet homme a pu alimenter autant la pensée libertine au XVIIle siècle que l'auteur des «Appareils idéologiques d'État» en passant par les éclairs nietzschéens et quelques travaux psychanalytiques du début du siècle. Lire Le Contrat social (plus ces étranges notes autographes du Projet de Constitution de la Corse) en biais avec cette randonnée botaniste apparemment différente, Les Rêveries du promeneuf solitaire. Lire la thèse de doctorat de Marx (genre académico-bourgeois par excellence) sur Démocrite et Épicure en rapport avec ce poème spectral qui dans sa langue défie toute traduction. Le Manifeste du Parti communiste - tel que déposé dans la livraison d'Études françaises (XVI), d'octobre 1980 sur le thème (aux incidences très québécoises) du "Manifeste poétique/politique». Et caetera...

Quel serait donc le lien entre tous ces modes d'exposition du désir politique, désir dont on ne peut faire l'économie de ses; origines révolutionnantes, ni méconnaître entre-temps la portée théorétique de son geste inaugural.

La pensée recueille la contradiction; en sa réflexivité et sur le territoire qui la marque de partout, elle recueille tous les paroxysmes issus de la contradiction. Et lorsqu'elle ne prend pas le détour parodique d'une philosophie d'électeurs et de faux retraités, elle redonne vie aux paroxysmes qui s'achèvent, 
s'effacent, s'annulent; elle pense néanmoins leur mort comme une nécessité déjà actualisée, offerte en dehors de tout régime de transcendance. Voilà pourquoi je dirais (en terminant) qu'une philosophie politique sur le mode pragmatico-desperado développe un Dire permanent qui invite les savoir-faire (militants ou non) à faire savoir les formes les plus quotidiennes, locales ici-maintenant, de l'exploitation et de l'oppression afin que les dénonçant, nous soyons rendus à l'indéfinie réflexion du désir et à la simplicité désarmante du mobile révolutionnaire.

Robert Hébert

Professeur au département de philosophie

Collège Maisonneuve 